\title{
The effect of aerobic exercise and vitamin D supplementation on inflammatory factors, anti- inflammatory proteins, and lung function in male smokers: A randomized controlled trial
}

\section{Leila Nikniaz}

Health Managment and Safety Promotion Research Institute

Morteza Ghojazadeh

Iranian EBM Center

Hooman Nateghian

Tabriz University of Medical Sciences

Mahdieh Abbasalizadeh Farhangi

Tabriz University of Medical Sciences

Hadi pourmanaf ( $\nabla$ hadipourmanaf@gmail.com )

Tehran University: University of Tehran https://orcid.org/0000-0001-7922-811X

\section{Research article}

Keywords: aerobic exercise, CC16, vitamin D, inflammation, SP-D, lung function, smoker

Posted Date: December 17th, 2020

DOI: https://doi.org/10.21203/rs.3.rs-126225/v1

License: (c) (i) This work is licensed under a Creative Commons Attribution 4.0 International License.

Read Full License 


\section{Abstract}

\section{Background}

This study aimed to investigate the effect of aerobic exercise and vitamin $D$ supplementation on inflammation (TNF-a, IL-6, CC16, SP-D, and CC16/SP-D ratio) and lung function (FEV 1, FVC, and $\mathrm{FEV}_{1} / \mathrm{FVC}$ ratio) in male smokers.

Methods

After applying inclusion criteria, a total of 40 healthy male smokers were recruited in this study. The subjects were randomly divided into four groups as follows: Aerobic Exercise + vitamin $D$ Supplementation ( $A E+V i t D, N=10)$, Aerobic Exercise ( $A E, N=10)$, vitamin $D$ Supplementation (VitD, $N=$ $10)$, and Control $(C, N=10)$. The subjects in the $A E+V i t D$ and $A E$ groups performed aerobic exercise training (running) up to $50 \%$ of heart rate (HR) max, three times a week for four weeks. Subjects in $A E+$ VitD and VitD groups received $6000 \mathrm{UI} / \mathrm{w}$ vitamin D3 for four weeks. The subjects in control group did not receive any intervention. Serum tumor necrosis factor (TNF)-a, interleukin (IL)-6, Clara cell protein (CC16), surfactant protein (SP)-D, CC16/SP-D ratio, and lung function (FEV1, FVC, and FEV1/FVC ratio) were measured before and after four weeks of intervention.

Results

Serum levels of TNF-a, IL-6, CC16, and SP-D decreased significantly in AE + VitD and AE groups after four weeks $(P<0.05)$, but serum SP-D level in the AE group did not decrease significantly. In addition, FEV1 and FVC increased significantly $(P<0.05)$ in AE + VitD and AE groups after four weeks of intervention. However, AE + VitD and AE did not have a significant effect on CC16/SP-D ratio and FEV1/FVC ratio. Furthermore, serum levels of TNF-a, IL-6, and CC16 decreased significantly after vitamin D supplementation $(P<0.05)$.

\section{Conclusions}

The results of present study demonstrates that aerobic exercise combined with vitamin $D$ supplementation can reduce inflammatory factors and anti-inflammatory proteins in the blood and improve lung function after four weeks. Further larger trials with higher sample size and longer duration are suggested to confirm these results.

Trial registration

Retrospectively registered. IRCT20180513039637N4. Registration date: 2020/10/20. URL: https://www.irct.ir/search/result?query=IRCT20180513039637N4

\section{Background}


Evidence suggests that tobacco-related diseases and deaths are growing in the world [1]. The most common type of tobacco use is cigarette smoking (CS) [2]. Long term cigarette smoking (LTCS) causes various diseases by affecting the organs of the body [3]. But the most well-known effect of toxic substances of cigarettes is on the respiratory system, which results in the pulmonary epithelial and cell membrane damages, and alter mucosal permeability $[4,5]$. Theses alterations cause such diseases as lung cancer and chronic obstructive pulmonary disease (COPD) $[3,6,7]$. Also, smoking reduces pulmonary function by decreasing $\mathrm{FEV}_{1}$ and FVC [8].

LTCS causes the release of inflammatory cells (e.g., macrophages, neutrophils, and lymphocytes) and inflammatory mediators (e.g., TNF-a and IL-6) from epithelial and smooth muscle cells and initiates the inflammatory process $[5,9,10]$. On the other hand, toxic substances pass through the alveolar and capillaries and enter the bloodstream. These toxins are identified by immune system receptors and trigger the inflammatory process via NF-kB in the bloodstream [5]. NF-kB has an important role in triggering inflammation by activating the expression of genes for inflammatory factors such as IL-6 and TNF- $a$ [11]. Also, lung epithelial cells release anti-inflammatory proteins such as Clara cell protein (CC16) and surfactant protein D (SP-D) during inflammation and disease $[12,13]$. Studies have also shown that CS increases serum levels of CC16 and SP-D $[13,14]$. Increasing CC16 and SP-D in serum and decreasing them in epithelial cells make lungs more vulnerable to damage [12]. In addition, the serum CC16/SP-D ratio is a valid and sensitive marker for the diagnosis of lung epithelial cell injury [12].

Previous studies have demonstrated the non-pharmacological effect of exercise training on reducing lung diseases $[5,15]$. Regular exercise can reduce inflammatory factors by activating the anti-inflammatory signaling pathway. This anti-inflammatory property of exercise training improves pulmonary rehabilitation, reduces lung diseases (e.g., dyspnea, airway hyperresponsiveness, exercise-induced bronchospasm, and asthma), and increases strength, endurance capacity, and quality of life $[5,15,16]$. Also, the results of Krüger et al. and Toledo-Arruda et al. showed that aerobic exercise can reduce CSinduced inflammation $[17,18]$. Furthermore, recent scientific evidence suggests that consumption of certain foods and nutrients improves lung function and reduces risks of COPD [11]. Interestingly, recent evidence demonstrates that vitamin $D$ [defined as 1,25-dihydroxyvitamin $D\left(1,25(\mathrm{OH})_{2} \mathrm{D}\right) \otimes 20 \mathrm{ng} / \mathrm{ml}$ ] deficiency causes lung disease and decrease lung function (FEV ${ }_{1}$ and FVC) [19]. Vitamin D is reported to have anti-inflammatory properties, and it can reduce CS-induced inflammation [19] by regulating the proliferation and function of immune cells through the vitamin D receptor (VDR), which is a member of the steroid hormone receptor family [20]. Agrawal et al. demonstrated that vitamin D supplementation can reduce the inflammatory effects [21].

Although some studies have confirmed the positive effects of aerobic exercise and vitamin D on reducing inflammation, few research has been conducted in this issue and the results are controversial. As far the researchers investigated, no study has examined the effect of aerobic exercise and vitamin $D$ supplementation on inflammation and lung function in tobacco smokers so far. Therefore, this study aimed at evaluating the effect of aerobic exercise and vitamin $D$ supplementation on inflammatory factors, anti-inflammatory proteins, and lung function in male smokers. 


\section{Methods}

In this experimental study, 40 healthy male smokers were recruited from Tabriz city, Iran. Inclusion criteria were as follows: at least one-year experience of smoking cigarettes, no prior history of specific diseases such as diabetes and cardiovascular and lung disease, nutritional allergies, and medication use, no prior history of using anti-inflammatory agents, $\beta_{2}$-agonists, any supplements (i.e. vitamins, proteins drinks, amino acids, etc.), or medication before or during the study, and no history of doing any regular exercise or physical activity in the past six months. Information on personal and family history of atopic diseases, diet, sociodemographic data, and frequency and duration of cigarette smoking were collected by a general questionnaire. After reviewing the personal and family questionnaire information and medical examinations, 40 healthy male smokers were selected. All subjects were informed about the objectives of the study and a written consent was obtained.

The study was approved by the Research Ethics Committee of Tabriz University of Medical Sciences, Iran which are in accordance with the Helsinki Declaration (Ethical code: IR.TBZMED.REC.1399.727). Also, the protocol of the study was registered in the Iranian registry of clinical trials (IRCT code: IRCT20180513039637N4).

Subjects were randomly divided into four groups: Aerobic Exercise + vitamin $D$ supplementation ( $A E+$ VitD, $N=10)$, Aerobic Exercise ( $A E, N=10)$, vitamin $D$ supplementation (VitD, $N=10)$, and Control $(C, N=$ 10). Randomization was carried out on an individual basis using Randomizer software. Opaque and sealed envelopes were used for allocation concealment. The groups were encoded, and the codes were sealed by an independent monitor.

All subjects were requested to follow their daily CS behavior and regular diet throughout the period of the study. Also, they were not allowed to use supplements such as omega-3 s that have anti-inflammatory properties [22]. Body weight (with $10 \mathrm{~g}$ measurement accuracy) was measured using a digital scale (OMRON, BF: 508, Finland). Body fat was measured using an Inbody 720 body Composition Analyzer with an accuracy of $0.5 \%$ (Inbody Co. Ltd.囚Seoul区 Korea). Body mass index (BMI) was calculated as weight in kilograms divided by height in meters squared, and Cooper test was used to estimate the maximum oxygen consumption $\left(\mathrm{VO}_{2} \mathrm{max}\right)$ of the subjects [23].

One week after the briefing session, the subjects began consumption of vitamin $D$ supplement and aerobic exercise. $A E+V i t D$ and $A E$ groups performed aerobic running for six weeks (30 minutes for three sessions a week) on the standard track racetrack. In the first three weeks, AE + VitD and AE groups ran at $50-60 \%$ of their individually determined maximum heart rate (HR). In the second three weeks of the study, the intensity and duration of the exercise increased to $60-70 \%$ HR max. POLAR (T31C, Finland) HR monitors were used to estimate subjects' HR and maintaining determined intensity during exercise. All exercise sessions were held between 8-10 am. Subjects in VitD and C groups were requested to perform their usual daily activities. Simultaneously with aerobic exercise, vitamin D3 supplementation started in VitD and $A E+$ VitD groups. Subjects in AE + VitD and VitD groups received $6000 \mathrm{UI} /$ week $(1000 \mathrm{IU} / \mathrm{day}=$ 
25 microgram, except Fridays) oral vitamin D3 in tablets form for six weeks. Tablets were produced and supplied by Health Aid Company (England).

To determine the baseline and final levels (after six weeks) of IL-6, TNF-a, CC16, SP-D, and 1,25dihydroxyvitamin $D(1,25(\mathrm{OH}) 2 \mathrm{D})$, blood samples were collected after 12-hour overnight fasting from the subjects' antecubital vein 24 hours before the start of the study $(10 \mathrm{ml})$ and 24 hours after the last aerobic exercise session ( $10 \mathrm{ml})$. Samples were centrifuged at 3,000 rpm for 15 minutes, and serum was subsequently distributed in 1.8-mL aliquots and stored at $-80^{\circ} \mathrm{C}$. IL-6, TNF-a, CC16, and SP-D were analyzed by ELISA using commercial kits (East Biopharm, USA).

FEV1 and FVC were measured before and after the study with an Easy One portable spirometer (ndd Medical Technologies, Zürich, Switzerland) following standard recommendations [24-26]. FEV1 and FVC were expressed as the percentage.

\section{Statistical analysis}

Statistical analysis was performed using SPSS (version 22). Descriptive data were reported as means and standard deviations. Normal distribution of data was verified with the Kolmogorov-Smirnov test. Paired sample t-test was used to compare within-group differences. Analysis of variance (ANOVA) and analysis of covariance (ANCOVA) were used to analyze between-group differences, respectively. If the value of F-ratio was significant, Bonferroni post hoc test was used to identify the differences between groups. P-value less than 0.05 was considered to be statistically significant.

\section{Results}

A total of 40 participants were recruited, with $91.6 \%$ of participants completing the study (Fig. 1; Consolidated Standards of Reporting Trials (CONSORT) diagram). The anthropometric characteristics, VO2 max, and duration of cigarette consumption are demonstrated in Table 1. Based on the results of one-way ANOVA, there was no significant difference in anthropometric characteristics, VO2 max, and cigarette consumption between the four groups $(P \otimes 0.05)$. 
Table 1

Anthropometric characteristics, VO2 max, and smoking duration in the studied groups

\begin{tabular}{|llllll|}
\hline Variables & AE + VitD $(n=10)$ & AE $(n=10)$ & VitD $(n=10)$ & $C(n=10)$ & $P D$ \\
\hline Age, year & $30.4 \pm 4.08$ & $31.3 \pm 4.00$ & $30.1 \pm 3.72$ & $31.7 \pm 3.83$ & 0.77 \\
\hline Height, $m$ & $1.74 \pm 0.06$ & $1.75 \pm 0.07$ & $1.74 \pm 0.09$ & $1.76 \pm 0.07$ & 0.96 \\
\hline Weight, kg & $76.00 \pm 9.00$ & $74.00 \pm 7.34$ & $75.10 \pm 9.1$ & $76.20 \pm 6.44$ & 0.92 \\
\hline BMl, kg.m ${ }^{2}$ & $24.90 \pm 1.57$ & $24.29 \pm 0.75$ & $25.19 \pm 3.47$ & $24.98 \pm 1.70$ & 0.80 \\
\hline Fat, \% & $14.50 \pm 2.54$ & $13.90 \pm 1.85$ & $14.90 \pm 2.51$ & $15.20 \pm 1.93$ & 0.59 \\
\hline VO $_{2}$ max, \% & $41.22 \pm 2.52$ & $40.28 \pm 3.25$ & $39.30 \pm 3.43$ & $40.40 \pm 2.41$ & 0.54 \\
\hline History of smoking, (year) & $6.90 \pm 2.37$ & $7.87 \pm 2.62$ & $7.65 \pm 3.10$ & $8.40 \pm 5.31$ & 0.85 \\
\hline Data are presented as Mean \pm SD & & & & \\
\hline 口 One-way ANOVA & & & & & \\
\hline
\end{tabular}

As Table 2 suggests, $\mathrm{AE}+\mathrm{VitD}(P \otimes 0.05), \mathrm{AE}(P \otimes 0.05)$, and VitD ( $P \otimes 0.05)$ resulted in a significant decrease in serum levels of TNF- $a$ and IL- 6 compared to the baseline, but the serum levels of TNF- $a$ and IL- 6 did not reduce in the control group ( $P \otimes 0.05)$. In addition, comparison of the mean group differences of TNF-a and IL- 6 showed no significant differences between the groups at the basic level and after the intervention (Table 2; P冈0.05). 
Table 2

Baseline and Week-4 values of inflammatory markers, anti-inflammatory proteins, lung function, and 1,25dihydroxy vitamin D

\begin{tabular}{|c|c|c|c|c|c|}
\hline Variables & $\begin{array}{l}A E+V i t D(n= \\
10)\end{array}$ & $\operatorname{AE}(n=10)$ & VitD $(n=10)$ & $C(n=10)$ & $\mathrm{Pl}$ \\
\hline \multicolumn{6}{|c|}{ TNF-a, $\left(\mathrm{ng} / \mathrm{ml}^{-1}\right)$} \\
\hline Baseline & $73.2 \pm 12.4$ & $75.4 \pm 15.0$ & $73.9 \pm 13.5$ & $74.1 \pm 10.6$ & 0.001 \\
\hline Week 4 & $55.0 \pm 11.4$ & $66.8 \pm 16.6$ & $67.7 \pm 11.1$ & $74.9 \pm 9.4$ & 0.001 \\
\hline$P^{\#}$ & 0.001 & 0.009 & 0.020 & 0.394 & \\
\hline \multicolumn{6}{|c|}{ IL-6, $\left(\mathrm{pg} / \mathrm{ml}^{-1}\right)$} \\
\hline Baseline & $5.87 \pm 1.61$ & $6.30 \pm 2.02$ & $5.95 \pm 2.11$ & $6.16 \pm 1.39$ & 0.001 \\
\hline Week 4 & $4.98 \pm 1.37$ & $5.93 \pm 1.67$ & $5.70 \pm 1.98$ & $6.31 \pm 1.37$ & 0.001 \\
\hline$P$ & 0.004 & 0.039 & 0.046 & 0.097 & \\
\hline \multicolumn{6}{|c|}{$\mathrm{CC} 16,\left(\mathrm{ng} / \mathrm{ml}^{-1}\right)$} \\
\hline Baseline & $7.19 \pm 1.28$ & $7.34 \pm 1.07$ & $7.24 \pm 1.81$ & $7.25 \pm 1.05$ & 0.001 \\
\hline Week 4 & $6.42 \pm 1.06$ & $7.11 \pm 1.02$ & $6.87 \pm 1.08$ & $7.34 \pm 0.97$ & 0.001 \\
\hline$P$ & 0.001 & 0.005 & 0.025 & 0.688 & \\
\hline \multicolumn{6}{|c|}{ SP-D, (ng.ml-1) } \\
\hline Baseline & $69.80 \pm 13.7$ & $71.83 \pm 12.9$ & $70.75 \pm 10.4$ & $71.20 \pm 11.5$ & 0.001 \\
\hline Week 4 & $62.06 \pm 9.4$ & $69.50 \pm 11.1$ & $68.4 \pm 8.3$ & $72.3 \pm 11.2$ & 0.001 \\
\hline$P$ & 0.011 & 0.085 & 0.084 & 0.240 & \\
\hline \multicolumn{6}{|c|}{ CC16/SP-D } \\
\hline Baseline & $0.104 \pm 0.014$ & $\begin{array}{l}0.102 \pm \\
0.010\end{array}$ & $\begin{array}{l}0.101 \pm \\
0.005\end{array}$ & $\begin{array}{l}0.102 \pm \\
0.009\end{array}$ & 0.001 \\
\hline Week 4 & $0.104 \pm 0.104$ & $\begin{array}{l}0.102 \pm \\
0.008\end{array}$ & $\begin{array}{l}0.099 \pm \\
0.005\end{array}$ & $\begin{array}{l}0.101 \pm \\
0.009\end{array}$ & \\
\hline$P$ & 0.817 & 0.810 & 0.393 & 0.903 & 0.856 \\
\hline \multicolumn{6}{|l|}{$\mathrm{FEV}_{1}, \%$} \\
\hline \multicolumn{6}{|c|}{ Data are presented as Mean \pm SD, } \\
\hline \multicolumn{6}{|c|}{ Q Inter-group comparisons with ANCOVA } \\
\hline \# paired s & & & & & \\
\hline
\end{tabular}




\begin{tabular}{|c|c|c|c|c|c|}
\hline Variables & $\begin{array}{l}A E+\operatorname{VitD}(n= \\
10)\end{array}$ & $\mathrm{AE}(n=10)$ & VitD $(n=10)$ & $C(n=10)$ & $\mathrm{Pl}$ \\
\hline Baseline & $87.29 \pm 7.51$ & $\begin{array}{l}88.30 \pm \\
10.54\end{array}$ & $87.55 \pm 8.48$ & $86.70 \pm 8.48$ & 0.001 \\
\hline Week 4 & $91.70 \pm 5.86$ & $91.80 \pm 8.23$ & $89.21 \pm 7.85$ & $\begin{array}{l}86.44 \pm \\
11.30\end{array}$ & 0.001 \\
\hline$P$ & 0.001 & 0.021 & 0.121 & 0.534 & \\
\hline \multicolumn{6}{|l|}{ FVC, \% } \\
\hline Baseline & $94.39 \pm 7.41$ & $\begin{array}{l}94.81 \pm \\
12.69\end{array}$ & $92.85 \pm 6.93$ & $\begin{array}{l}93.15 \pm \\
10.10\end{array}$ & 0.001 \\
\hline Week 4 & $97.97 \pm 6.94$ & $97.65 \pm 9.51$ & $93.95 \pm 7.99$ & $\begin{array}{l}92.55 \pm \\
10.73\end{array}$ & 0.004 \\
\hline$P$ & 0.002 & 0.035 & 0.313 & 0.133 & \\
\hline \multicolumn{6}{|c|}{$\mathrm{FEV}_{1} / \mathrm{FVC}, \%$} \\
\hline Baseline & $92.45 \pm 2.59$ & $93.42 \pm 3.97$ & $94.20 \pm 4.08$ & $92.89 \pm 3.59$ & 0.001 \\
\hline Week 4 & $93.78 \pm 3.71$ & $94.17 \pm 3.99$ & $95.06 \pm 4.11$ & $93.31 \pm 4.22$ & 0.934 \\
\hline$P$ & 0.153 & 0.397 & 0.643 & 0.478 & \\
\hline \multicolumn{6}{|c|}{$\begin{array}{l}1,25(\mathrm{OH})_{2} \mathrm{D},\left(\mathrm{ng} / \mathrm{ml}^{-}\right. \\
\left.{ }^{1}\right)\end{array}$} \\
\hline Baseline & $23.90 \pm 5.72$ & $23.38 \pm 6.65$ & $24.51 \pm 7.82$ & $24.73 \pm 7.60$ & 0.001 \\
\hline Week 4 & $27.48 \pm 6.85$ & $24.15 \pm 5.51$ & $27.55 \pm 8.05$ & $24.64 \pm 7.18$ & 0.004 \\
\hline$P$ & 0.002 & 0.237 & 0.001 & 0.924 & \\
\hline \multicolumn{6}{|c|}{ Data are presented as Mean $\pm S D$, } \\
\hline \multicolumn{6}{|c|}{ I Inter-group comparisons with ANCOVA } \\
\hline \# paired sa & & & & & \\
\hline
\end{tabular}

As shown in Table 2, there was a significant difference between groups in CC16 and SP-D values before (baseline) and after (four weeks) the intervention (P凶0.05). In addition, inter-group difference of CC16/SP$D$ ratio at the baseline was significant $(P \otimes 0.05)$; however, no significant difference was observed between groups after four weeks $(P \Downarrow 0.05) . \mathrm{AE}+\operatorname{VitD}(P=0.001), \mathrm{AE}(P=0.005)$, and VitD $(P=0.025)$ reduced the levels of CC16 compared to baseline values; but no significant intra-group difference in the CC16/SP-D ratio was observed between the studied groups. Furthermore, serum SP-D level decreased significantly only in the $\mathrm{AE}+\operatorname{VitD}(P=0.011)$ group and no significant difference was observed in any of the groups (Table 2; P凶0.05). 
According to the results, after four weeks of intervention, $\operatorname{FEV}_{1}(P=0.001)$ and $\operatorname{FVC}(P=0.001)$ significantly increased in the $A E+V i t D$ and $A E$ groups, but none of the interventions had a significant effect on the $\mathrm{FEV}_{1} / \mathrm{FVC}$ ratio ( $\left.P \otimes 0.05\right)$. In addition, a significant difference was observed between the groups in the amount of $\mathrm{FEV}_{1}$ and $\mathrm{FVC}$ before and after the intervention ( $\left.P \otimes 0.05\right)$. Also, inter-group difference of $\mathrm{FEV}_{1} / \mathrm{FVC}$ ratio at the baseline was significant ( $\left.P \otimes 0.05\right)$; but no significant difference was observed between groups after four weeks $(P \otimes 0.05)$

Furthermore, changes of serum 1,25-dihydroxyvitamin $D\left(1,25(\mathrm{OH})_{2} \mathrm{D}\right)$ were significant between the groups ( $P \otimes 0.05)$. Also, the serum levels of 1,25-dihydroxyvitamin $\mathrm{D}(P=0.001)$ increased only in the $\mathrm{AE}+$ VitD and VitD groups; but $\mathrm{AE}(P=0.41)$ and $\mathrm{AE}+\operatorname{VitD}(P=0.86)$ groups did not have an effect on serum levels of 1,25-dihydroxyvitamin $D$.

\section{Discussion}

In the present study, AE + VitD, AE, and VitD reduced inflammatory factors )TNF-a and IL-6 (in the bloodstream, but no significant difference was observed in the control group after four weeks. Previous studies have demonstrated that physical activity $[7,18]$ and vitamin $D$ supplementation $[19,20]$ can reduce inflammatory cytokines. Exercise training moderates inflammatory process by activating antiinflammatory signaling pathways [5]. Exercise training inserts its anti-inflammatory properties by increasing systemic levels of anti-inflammatory cytokines such as IL-1RA, and prevents the secretion of pro-inflammatory factors [5]. On the other hand, prolonged exercise plays an important role in reducing the production of the pro-inflammatory factors by decreasing expression of Toll-like receptors (TLRs) on monocytes [5]. In addition, vitamin D supplementation reduces inflammation by inhibiting the proliferation of T-cells and the production of cytokines [20].

The results of this study showed that $A E+V i t D, A E$, and VitD can reduce CC16 after four weeks, but only $A E+V i t D$ could reduce serum SP-D levels after four weeks. Furthermore, interventions did not have a significant effect on CC16/SP-D ratio. CC16 is used as a marker of distal lung epithelial damage [27]. Although biological function of CC16 has not been completely discovered, it seems that it can also have direct anti-inflammatory properties through inhibiting the enzyme phospholipase $A_{2}$ and the protein prostaglandin $\mathrm{D}_{2}$ release, which both are involved in the arachidonic acid inflammatory cascade [28]. Also, SP-D is a protein in pulmonary host defense that is released from the epithelial cells of the lungs to interact with alveolar macrophages and a variety of microorganisms $[13,29,30]$. We did not find any research on the effect of aerobic exercise and vitamin D supplementation on serum CC16 and SP-D levels. Only in one research, Moazami et al. reported a significant reduction in serum levels of CC16 after four weeks of aerobic exercise with methadone [31].

Based on the results of this study, $A E+$ VitD and $A E$ significantly increased $F E V_{1}$ and $F V C$ after four weeks in male smokers. According to the results, physical activity can improve lung function [32-34]. Garcia et al. reported that moderate to high levels of regular exercise can increase lung function in smokers [35]. It seems that regular exercise prevents lung function decline by reducing inflammatory markers and 
oxidative, and it can have a positive effect on respiratory muscle strength [35]. On the other hand, prior studies have shown an association between vitamin $D$ and lung function. Vitamin $D$ sufficiency can protect lung function against the inflammatory and oxidative effects of smoking [36]. However, the results of the present study showed that vitamin D supplementation had no effect on improving lung function. We speculate that four weeks of supplementation is not enough to see the positive effects on lung function. So, studies with longer durations are needed.

This is the first study that examined the effect of aerobic exercise and vitamin D supplementation on inflammatory and anti-inflammatory agents and pulmonary function. The results of this study should be interpreted considering the limitations. We did not study vitamin $D$ supplementation and aerobic activity over longer periods of time (e.g., in 8-12 weeks).

In conclusion, the results of present study demonstrated that the interaction of aerobic exercise (running with intensity of more than $50 \% \mathrm{HR}$ max for four weeks) and vitamin D supplementation $(6,000 \mathrm{UI} /$ week $)$ could reduce inflammatory factors, increase anti-inflammatory proteins, and improve lung function in male smokers. Further larger trials with higher sample size and longer duration are suggested to confirm these results.

\section{Abbreviations}

Aerobic Exercise + vitamin D Supplementation (AE + VitD)

Aerobic Exercise (AE)

Vitamin D Supplementation (VitD)

Control (C)

Heart Rate (HR)

Tumor Necrosis Factor-a (TNF-a)

Interleukin-6 (IL-6)

Clara Cell Protein (CC16)

Surfactant Protein-D (SP-D)

Forced Expiratory Volume in one second $\left(\mathrm{FEV}_{1}\right)$

Forced Vital Capacity (FVC)

Cigarette Smoking (CS)

Long Term Cigarette Smoking (LTCS) 
Chronic Obstructive Pulmonary Disease (COPD)

1,25-dihydroxyvitamin $\mathrm{D}\left(1,25(\mathrm{OH})_{2} \mathrm{D}\right)$

Vitamin D Receptor (VDR)

Body Mass Index (BMI)

Maximum oxygen consumption $\left(\mathrm{VO}_{2} \max \right)$

Analysis of Variance (ANOVA)

Analysis of Covariance (ANCOVA)

Toll-Like Receptors (TLRs).

\section{Declarations}

\section{Ethics approval and consent to participate:}

The protocol of the study was approved by the ethics committee of Tabriz university of medical sciences in accordance with the Helsinki Declaration (Ethical code: IR.TBZMED.REC.1399.727). A written consent was obtained from participants.

\section{Consent to publish}

Not applicable

\section{Availability of data and materials}

If requested, it will be available

\section{Competing interests}

No competing interests

\section{Funding}

This research received no specific grant from any funding agency.

\section{Authors' contributions}

H.P and M.A designed the project and aided in interpreting the results; L.N directed the project, developed the theoretical framework, and drafted the manuscript; M.G performed the analysis, designed the figures and interpreted the results; H.P, H.N and L.N wrote the manuscript. All authors provided critical feedback and helped shape the research, analysis and manuscript. 
Acknowledgment: The authors wish to thank the Student Research Committee for approving and supporting the project.

\section{References}

1. Organization, W.H., The world health report 2002: reducing risks, promoting healthy life. 2002.

2. Ng, M., et al., Smoking prevalence and cigarette consumption in 187 countries, 1980-2012. Jama, 2014. 311(2): p. 183-192.

3. Yanbaeva, D.G., et al., Systemic effects of smoking. Chest, 2007. 131(5): p. 1557-1566.

4. Čužić, S., et al., Claudin-3 and Clara Cell 10 kDa Protein as Early Signals of Cigarette Smoke-Induced Epithelial Injury along Alveolar Ducts. Toxicologic pathology, 2012. 40(8): p. 1169-1187.

5. Madani, A., et al., Immune-regulating effects of exercise on cigarette smoke-induced inflammation. Journal of inflammation research, 2018. 11: p. 155.

6. Hoseini, M.A.S., et al., Acute and Recovery Changes of TNF- $a$ and IL-1 $\beta$ in Response to Aerobic Exercise in Smokers and Non-smokers. International Journal of Basic Science in Medicine, 2018. 3(3): p. 109-113.

7. Krüger, K., et al., Time course of cigarette smoke-induced changes of systemic inflammation and muscle structure. American Journal of Physiology-Lung Cellular and Molecular Physiology, 2015. 309(2): p. L119-L128.

8. Allinson, J.P., et al., Combined impact of smoking and early-life exposures on adult lung function trajectories. American journal of respiratory and critical care medicine, 2017. 196(8): p. 1021-1030.

9. Alexander, L.E.C., S. Shin, and J.H. Hwang, Inflammatory diseases of the lung induced by conventional cigarette smoke: a review. Chest, 2015. 148(5): p. 1307-1322.

10. Kou, Y.R., K. Kwong, and L.-Y. Lee, Airway inflammation and hypersensitivity induced by chronic smoking. Respiratory physiology \& neurobiology, 2011. 178(3): p. 395-405.

11. Scoditti, E., et al., Role of diet in chronic obstructive pulmonary disease prevention and treatment. Nutrients, 2019. 11(6): p. 1357.

12. Combes, A., et al., Continuous exercise induces airway epithelium damage while a matched-intensity and volume intermittent exercise does not. Respiratory research, 2019. 20(1): p. 1-9.

13. Winkler, C., et al., Comprehensive characterisation of pulmonary and serum surfactant protein D in COPD. Respiratory research, 2011. 12(1): p. 1-11.

14. Knabe, L., et al., Club cells and CC16: another "smoking gun"?(With potential bullets against COPD). 2015, Eur Respiratory Soc.

15. Silva, R.A., et al., Aerobic training reverses airway inflammation and remodelling in an asthma murine model. European Respiratory Journal, 2010. 35(5): p. 994-1002.

16. Fanelli, A., et al., Exercise training on disease control and quality of life in asthmatic children. Medicine \& Science in Sports \& Exercise, 2007. 39(9): p. 1474-1480. 
17. Krüger, K., et al., Exercise training reverses inflammation and muscle wasting after tobacco smoke exposure. American Journal of Physiology-Regulatory, Integrative and Comparative Physiology, 2018. 314(3): p. R366-R376.

18. Toledo-Arruda, A.C., et al., Time-course effects of aerobic physical training in the prevention of cigarette smoke-induced COPD. Journal of applied physiology, 2017. 123(3): p. 674-683.

19. Heulens, N., et al., Vitamin D deficiency exacerbates COPD-like characteristics in the lungs of cigarette smoke-exposed mice. Respiratory research, 2015. 16(1): p. 110.

20. Matheu, V., et al., Dual effects of vitamin D-induced alteration of TH1/TH2 cytokine expression: enhancing IgE production and decreasing airway eosinophilia in murine allergic airway disease. Journal of allergy and clinical immunology, 2003. 112(3): p. 585-592.

21. Agrawal, T., G.K. Gupta, and D.K. Agrawal, Vitamin D supplementation reduces airway hyperresponsiveness and allergic airway inflammation in a murine model. Clinical \& Experimental Allergy, 2013. 43(6): p. 672-683.

22. Rowe, B. and L. Davis, Anti-inflammatory Foods for Health: Hundreds of Ways to Incorporate Omega3 Rich Foods Into Your Diet to Fight Arthritis, Cancer, Heart Disease, and More. 2008: Fair Winds Press (MA).

23. Cooper, S. and C.E. Helmstetter, Chromosome replication and the division cycle of Escherichia coli Br. Journal of molecular biology, 1968. 31(3): p. 519-540.

24. Bolger, C., et al., Hyperpnea-induced bronchoconstriction and urinary CC16 levels in athletes. Medicine and science in sports and exercise, 2011. 43(7): p. 1207-1213.

25. Miller, M., et al., col.(2005). Standardisation of spirometry. European Respiratory Journal. 26(2): p. 319-38.

26. Tufvesson, E., et al., Increase of club cell (Clara) protein (CC16) in plasma and urine after exercise challenge in asthmatics and healthy controls, and correlations to exhaled breath temperature and exhaled nitric oxide. Respiratory medicine, 2013. 107(11): p. 1675-1681.

27. Chimenti, L., et al., Bronchial epithelial damage after a half-marathon in nonasthmatic amateur runners. American Journal of Physiology-Lung Cellular and Molecular Physiology, 2010. 298(6): p. L857-L862.

28. Ma, Y.-N., et al. Association of urine CC16 and lung function and asthma in Chinese children. in Allergy \& Asthma Proceedings. 2015.

29. Matalon, S., et al., Modification of surfactant protein D by reactive oxygen-nitrogen intermediates is accompanied by loss of aggregating activity, in vitro and in vivo. The FASEB Journal, 2009. 23(5): p. 1415-1430.

30. Lomas, D., et al., Serum surfactant protein D is steroid sensitive and associated with exacerbations of COPD. European respiratory journal, 2009. 34(1): p. 95-102.

31. Moazami, M., et al., The effect of aerobic training on Clara Cell protein 16 (CC16) and cortisol in addicts. Razi Jou of Med Sci, 2015. 1(22): p. 132-140. 
32. Azad, A., et al., Effects of aerobic exercise on lung function in overweight and obese students. Tanaffos, 2011. 10(3): p. 24.

33. Cielen, N., et al., Interaction between physical activity and smoking on lung, muscle, and bone in mice. American Journal of Respiratory Cell and Molecular Biology, 2016. 54(5): p. 674-682.

34. Hulke, S.M., Effect of endurance training on lung function: a longitudinal study. Int J Biol Med Res, 2011. 2(1): p. 443-446.

35. Garcia-Aymerich, J., et al., Regular physical activity modifies smoking-related lung function decline and reduces risk of chronic obstructive pulmonary disease: a population-based cohort study. American journal of respiratory and critical care medicine, 2007. 175(5): p. 458-463.

36. Lange, N.E., et al., Vitamin D deficiency, smoking, and lung function in the Normative Aging Study. American journal of respiratory and critical care medicine, 2012. 186(7): p. 616-621.

\section{Figures}




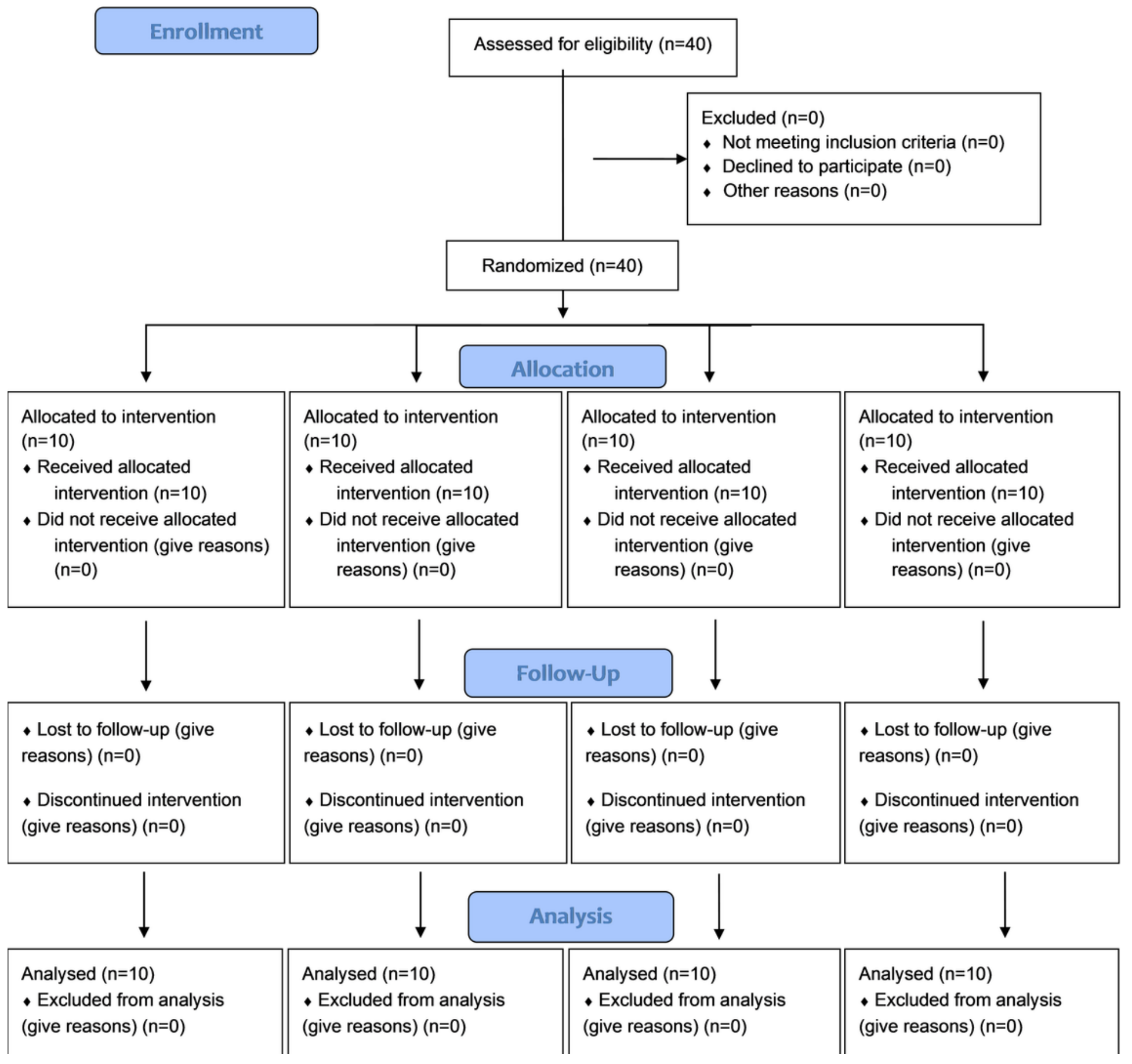

\section{Figure 1}




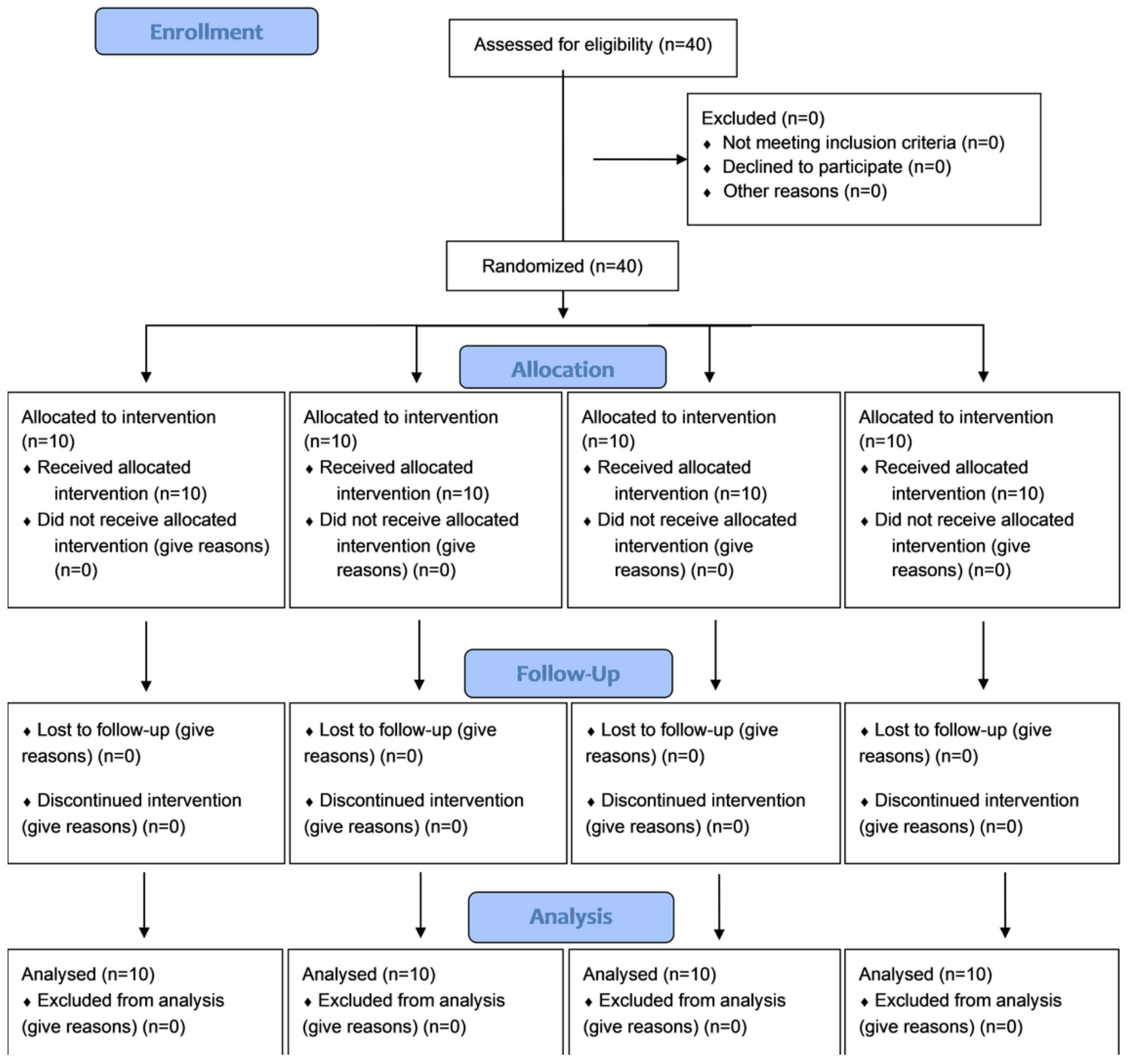

\section{Figure 1}




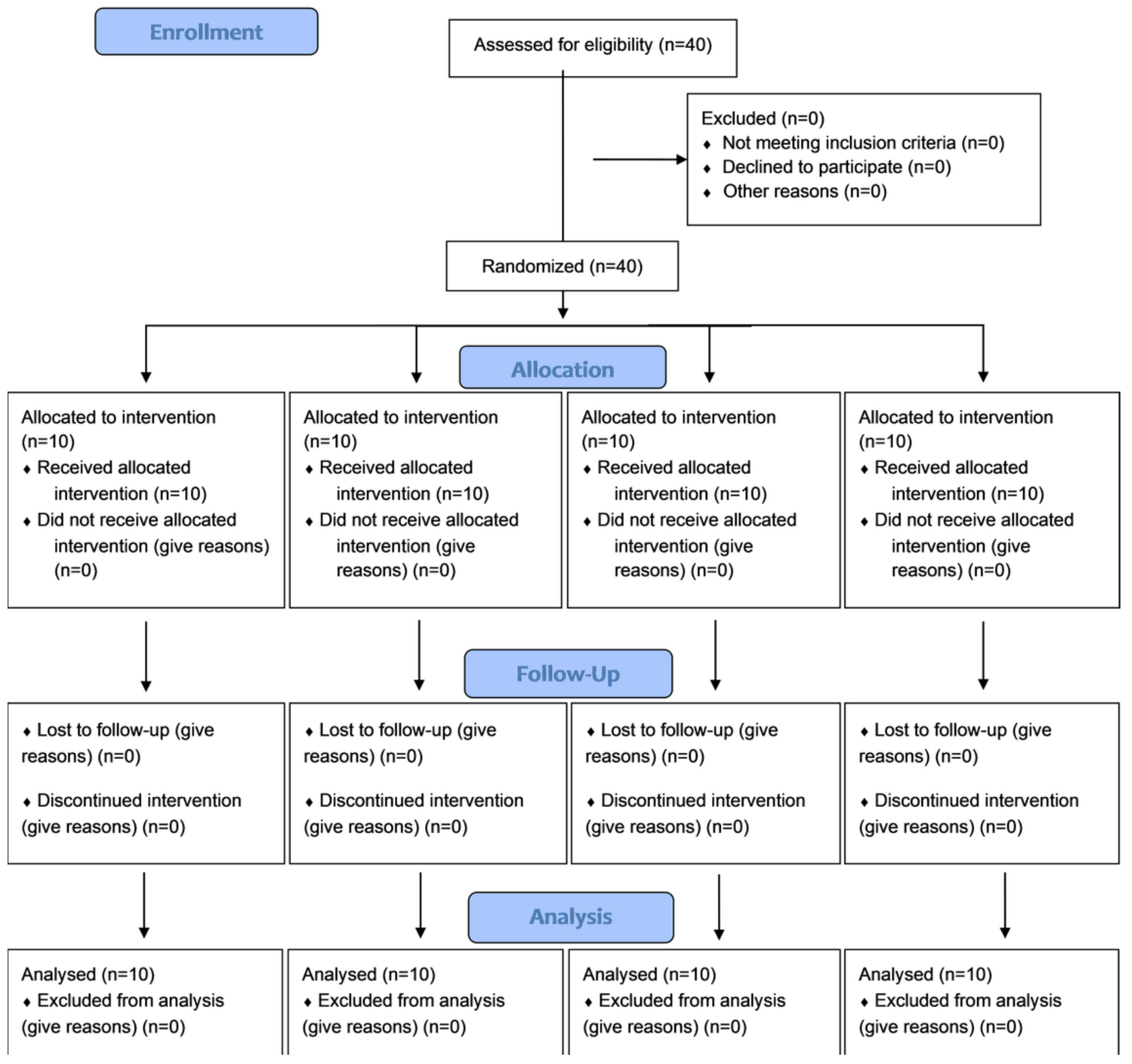

\section{Figure 1}




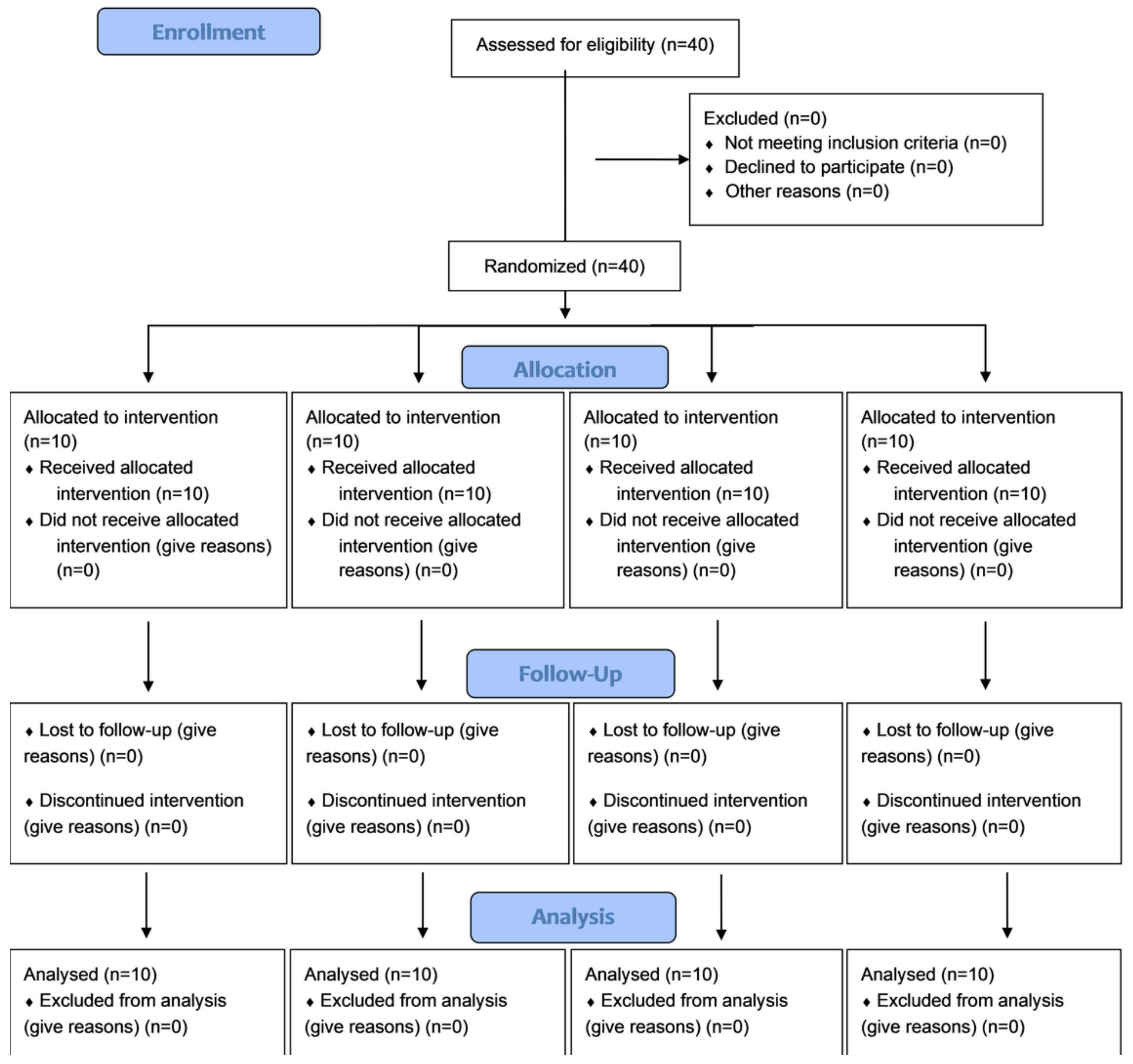

\section{Figure 1}

\section{Supplementary Files}

This is a list of supplementary files associated with this preprint. Click to download. 
- CONSORTstatement.doc

- CONSORTstatement.doc 